\title{
Efficacy of temozolomide and bevacizumab for the treatment of leptomeningeal dissemination of recurrent glioblastoma: A case report
}

\author{
YOSHIKO OKITA $^{1}$, MASAHIRO NONAKA ${ }^{1}$, TORU UMEHARA ${ }^{1}$, YONEHIRO KANEMURA ${ }^{1,2}$, \\ YOSHINORI KODAMA $^{3}$, MASAYUKI MANO ${ }^{3}$ and SHIN NAKAJIMA ${ }^{1}$ \\ ${ }^{1}$ Department of Neurosurgery, ${ }^{2}$ Division of Regenerative Medicine, Institute for Clinical Research and \\ ${ }^{3}$ Department of Central Laboratory and Surgical Pathology, Osaka National Hospital, \\ National Hospital Organization, Osaka 540-0006, Japan
}

Received May 26, 2014; Accepted January 19, 2015

DOI: $10.3892 / \mathrm{ol} .2015 .2940$

\begin{abstract}
The prognosis of leptomeningeal dissemination of recurrent glioblastoma is poor, and chemotherapy results in minimal palliative efficacy. Temozolomide (TMZ) is an established therapy for patients with malignant glioma and the standard of care in parenchymal gliomas; however, few reports have been published with regard to its use for the treatment of leptomeningeal dissemination. Only one report has indicated the radiographic response of leptomeningeal dissemination to a TMZ rechallenge, suggesting a potential causative effect. While bevacizumab is an effective therapy for recurrent glioblastoma, its effect on leptomeningeal dissemination of recurrent glioblastoma remains unclear. The present study reports a case of leptomeningeal dissemination of recurrent glioblastoma in which transient neurological and radiological improvement was observed following chemotherapy with TMZ and bevacizumab. However, five months after the diagnosis of leptomeningeal dissemination the patient succumbed to the disease.
\end{abstract}

\section{Introduction}

Glioblastomas are the most common type of common primary intracranial tumor. The prognosis of recurrent glioblastoma is poor with a patient survival time of between one and two years. Temozolomide (TMZ) is the standard therapy for glioblastoma patients. A recent study revealed an improvement in the median survival time from 12.1 months to 14.6 months upon the addition of concurrent TMZ to the previous standard therapy of surgery

Correspondence to: Dr Masahiro Nonaka, Department of Neurosurgery, Osaka National Hospital, National Hospital Organization, 2-1-14 Hoenzaka, Osaka 540-0006, Japan

E-mail: nonakamasa65@gmail.com

Key words: leptomeningeal dissemination, recurrent glioblastoma, bevacizumab and radiotherapy in glioblastoma patients (1). In cases of recurrence, the median survival is approximately six months $(2,3)$. Leptomeningeal dissemination, an end-stage complication of glioblastoma, is considered to be untreatable, with reported mean survival times of two to four months (4-6). Furthermore, previous reports have indicated that chemotherapy for leptomeningeal dissemination has limited therapeutic efficacy $(4,7)$.

The utility of TMZ for the treatment of leptomeningeal dissemination of recurrent malignant glioma has not been determined, with only one study reporting a radiographic response to a TMZ rechallenge, which indicates a potential causative effect (8). Nandipati et al (8) presented the cases of two anaplastic glioma patients who exhibited durable responses of leptomeningeal dissemination to TMZ treatment. One patient received 15 TMZ chemotherapy cycles $(200 \mathrm{mg}$ / $\mathrm{m}^{2} /$ day for 5 days in a 28 day cycle) in total with radiographic improvement after four cycles and resolution following six cycles of TMZ. The patient remains alive 34 months following the diagnosis of leptomeningeal dissemination. The second patient was treated with radiation therapy without concurrent chemotherapy and then a subsequent 12 cycles of TMZ chemotherapy were administered. All enhanced disease sites disappeared after six cycles. Nine months following the discontinuation of chemotherapy, neuroimaging revealed multiple, asymptomatic, enhanced nodules involving the leptomeninges. The patient received additional TMZ chemotherapy and all lesions disappeared after an additional six cycles. Forty-eight months following the initial diagnosis of leptomeningeal dissemination, the patient demonstrates no neurological deficit on TMZ (chemotherapy was ongoing at the final follow-up in 2010).

Bevacizumab, a monoclonal antibody that inhibits vascular endothelial growth factor (VEGF), is an effective established therapy for recurrent glioblastoma, following treatment with radiotherapy plus TMZ (9). Bevacizumab is also approved for the treatment of metastatic colorectal, non-small-cell lung, breast, ovarian and renal cancers. Although a pilot study demonstrated some response of stable disease to bevacizumab in a small sample of patients with leptomeningeal metastases from breast and lung cancers, in addition to melanoma (10), only 
A

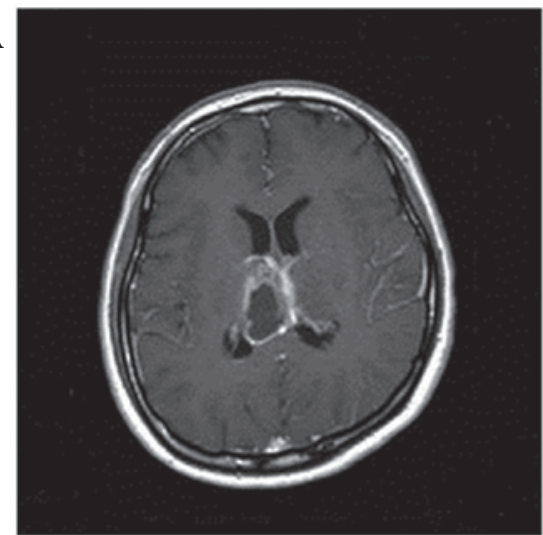

C

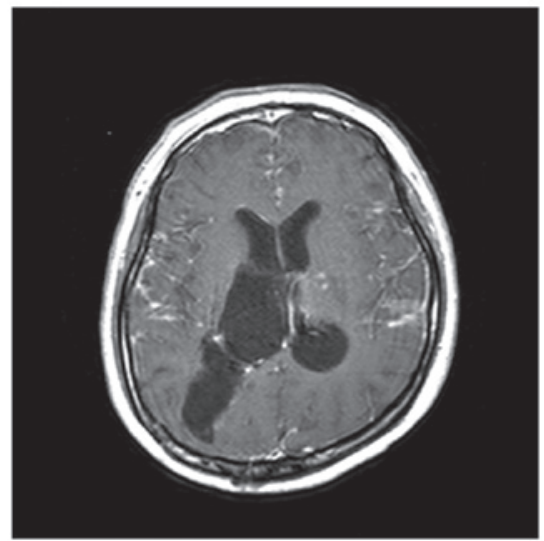

B

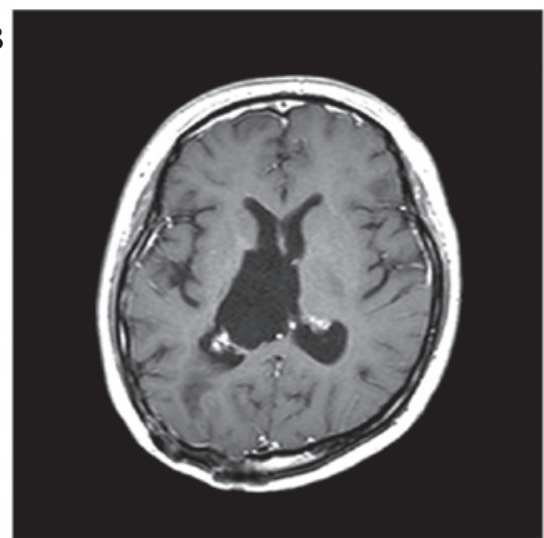

D

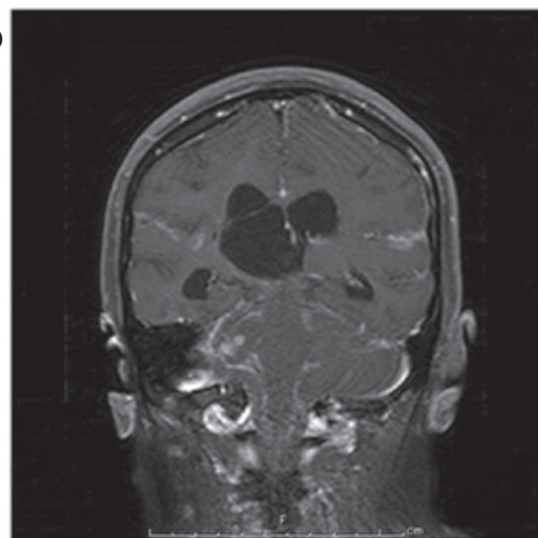

Figure 1. (A) MRI prior to surgery showed a tumor in the right thalamus enhanced with gadolinium diethylenetriamine pentaacetic acid. (B) MRI following maintenance TMZ therapy showed the enhanced lesion in the right thalamus had disappeared without recurrence. (C and D) Recurrent tumor was revealed on MRI; leptomeningeal dissemination was identified in the supra- and infratentorial brain without primary site recurrence. MRI, magnetic resonance imaging; TMZ, temozolamide.

one study has examined the effect on leptomeningeal dissemination of glioblastoma, and no improvement was observed (11).

The current study reports the case of one patient treated with TMZ and bevacizumab for leptomeningeal dissemination of recurrent glioblastoma. Written informed consent was obtained from the patient.

\section{Case report}

A 28-year-old female with glioblastoma was admitted to the Department of Neurosurgery, Osaka National Hospital (Osaka, Japan) after presenting with diplopia and numbness in the left hand. Magnetic resonance imaging (MRI) revealed an enhanced mass in the right thalamus (Fig. 1A). The tumor was surgically removed, and the histological diagnosis was determined to be glioblastoma. Radiotherapy $(60 \mathrm{~Gy} / 30 \mathrm{fr})$ with concurrent TMZ ( $75 \mathrm{mg} / \mathrm{m}^{2}$ per day) was administered for a month and a half, following which the patient was discharged from the hospital. A further 24 cycles of outpatient maintenance chemotherapy with TMZ were administered: First cycle, $150 \mathrm{mg} / \mathrm{m}^{2}$; second cycle onwards, $200 \mathrm{mg} / \mathrm{m}^{2}$, for the first five days of each 28 -day cycle. No complications or recurrence were observed during maintenance chemotherapy (Fig. 1B). However, two months following the completion of the maintenance $\mathrm{TMZ}$ therapy, the patient experienced a seizure and disturbed consciousness. MRI revealed leptomeningeal dissemination in the supra- and infratentorial brain without primary site recurrence (Fig. 1C and D). Cerebrospinal fluid (CSF) cytology specimens were positive for malignant cells. Following one cycle of combined TMZ $\left(150 \mathrm{mg} / \mathrm{m}^{2}\right)$ and interferon- $\beta$ therapy (3 MU) for the first five days of a 28 -day cycle, MRI indicated progression of the leptomeningeal dissemination (Fig. 2A and B).

TMZ $\left(150 \mathrm{mg} / \mathrm{m}^{2}\right)$ for the first five days of a 28 -day cycle and bevacizumab $(10 \mathrm{mg} / \mathrm{kg})$ for every day of a two-week cycle were administered. Following two cycles of TMZ and one cycle of bevacizumab from the beginning of recurrent therapy, the leptomeningeal dissemination observed on MRI decreased (Fig. 2C and D). The patient's consciousness improved marginally, and she was gradually able to maintain simple communication. Following three cycles each of TMZ and bevacizumab from the beginning of recurrent therapy, MRI showed no deterioration of leptomeningeal dissemination (Fig. 2E and F). However, following four cycles of TMZ and five cycles of bevacizumab from the beginning of recurrent therapy, progression of the leptomeningeal dissemination was observed (Fig. 3), and the patient became bedridden. Palliative treatment was administered, and the patient succumbed to the disease progression one month after the TMZ and bevacizumab therapy was discontinued.

\section{Discussion}

Leptomeningeal dissemination occurs in $\leq 20 \%$ of supra- and infratentorial glioblastoma cases, as described in several 
A

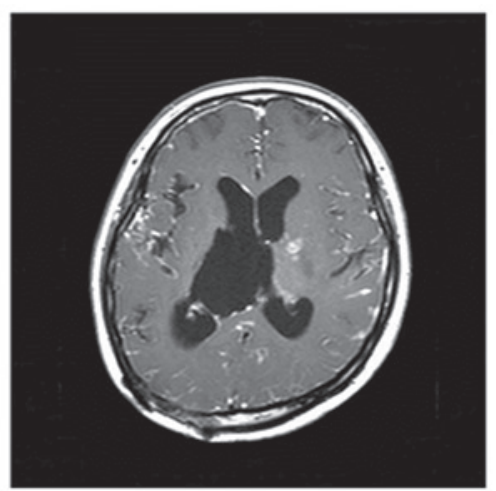

$\mathbf{B}$

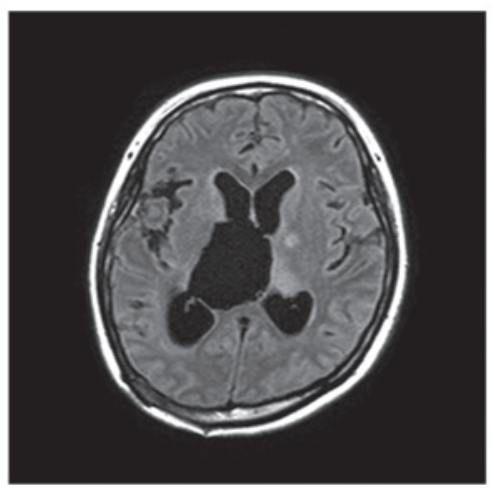

C

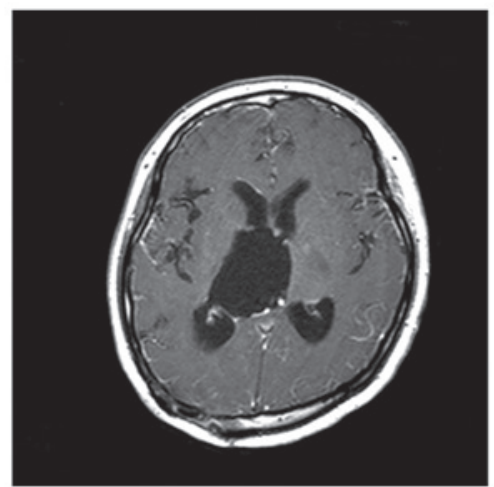

D

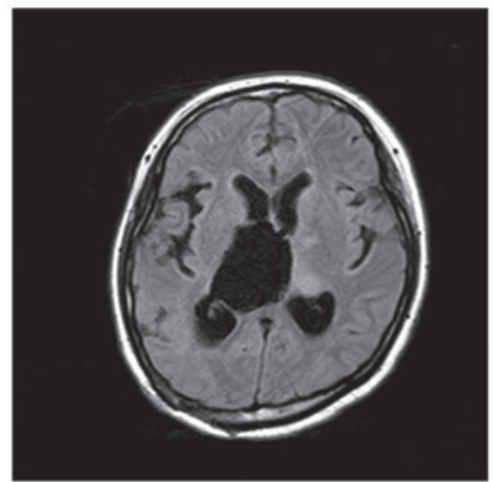

$\mathbf{E}$

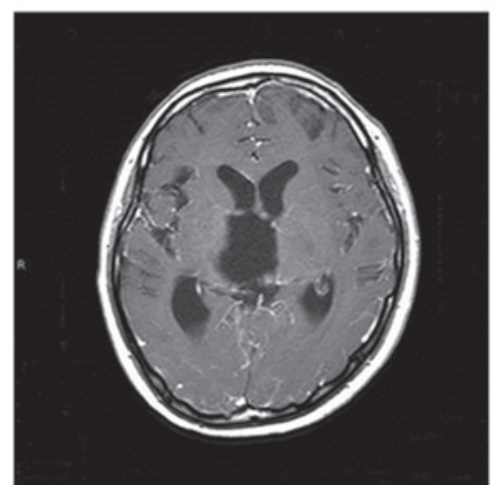

$\mathbf{F}$

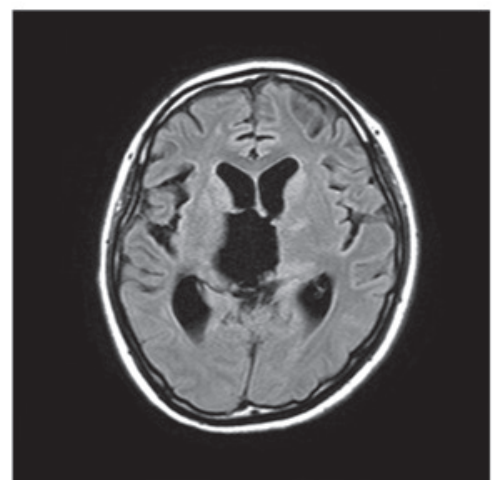

Figure 2. (A, B) MRI showed the progression of leptomeningeal dissemination following one cycle of TMZ and interferon- $\beta$ therapy. (C, D) Leptomeningeal dissemination on MRI decreased following two cycles of TMZ and one cycle of bevacizumab from the beginning of recurrent therapy. (E, F) MRI showed no progression of leptomeningeal dissemination following three cycles each of TMZ and bevacizumab from the beginning of recurrent therapy. A, C and E: T1-weighted contrast-enhanced MRI; B, D and F: Fluid-attenuated inversion recovery imaging. MRI, magnetic resonance imaging; TMZ, temozolomide.
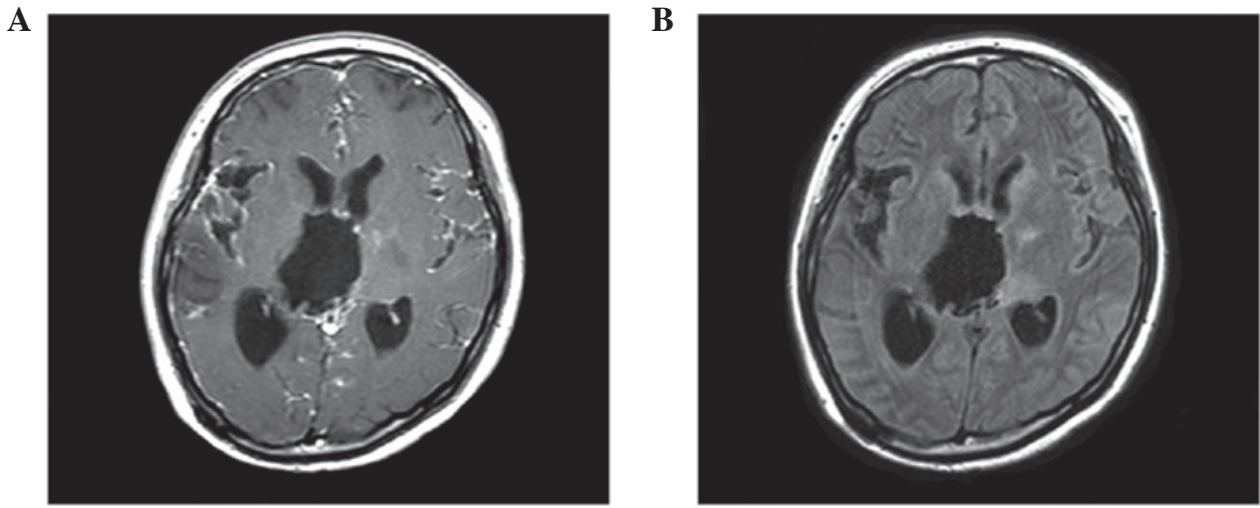

Figure 3. MRI showed progression of leptomeningeal dissemination following four cycles of TMZ and five cycles of bevacizumab from the beginning of recurrent therapy. (A) T1-weighted contrast-enhanced MRI; (B) fluid-attenuated inversion recovery imaging. MRI, magnetic resonance imaging; TMZ, temozolamide.

autopsy studies $(4,12,13)$, with a mean survival time of two to four months (4-6). Radiotherapy and chemotherapy result in minimal palliative efficacy, without increased survival $(6,14)$.

Bevacizumab is a humanized monoclonal antibody against VEGF, which has demonstrated improved progression-free survival (PFS) in phase III trials of metastatic breast (15) and renal cancers (16), in addition to prolonged overall survival in metastatic colorectal $(17,18)$ and non-small cell lung cancers (19). Bevacizumab has also been used as a successful therapy in cases of leptomeningeal metastases from breast (20), non-small cell lung (21), and colorectal cancers (22). Furthermore, a pilot study $(n=15)$ indicated that bevacizumab significantly decreases CSF
VEGF levels over time, resulting in clinical, imaging and CSF responses, or stable disease in $54-73 \%$ of leptomeningeal metastases patients with breast cancer, lung cancer, or melanoma $(10,23)$.

Chemotherapy as second- or third-line metastatic treatment has been established for other types of cancer, including breast and colorectal cancer $(24,25)$. While the blood-brain barrier is disrupted by metastases, bevacizumab may aid in normalizing this disrupted tumor vasculature and improve drug delivery by facilitating uniform distribution of the vasculature (26-28). As a result, the use of bevacizumab has aided in prolonging the response to second- or third-line metastatic treatment in other types of cancer (20). 
Other than TMZ or bevacizumab, no effective agent is available to treat glioblastoma. Bevacizumab alone or in combination with irinotecan was similarly effective for recurrent glioblastoma in the BRAIN study (29). Wong et al (30) reported that the six-month PFS rate and overall survival following bevacizumab therapy for recurrent glioblastoma were $45 \%$ and 9.3 months, respectively. However, the role of bevacizumab in the treatment of leptomeningeal dissemination of recurrent glioblastoma remains unclear due to the limited number of reported cases. The one previously published study did not report any improvement with this treatment (11).

TMZ is the standard therapy for patients with malignant glioma (1) and is considered the standard of care in parenchymal gliomas, but little published information exists with regard to the use of this agent to treat leptomeningeal dissemination. Nandipati et al (8) reported a radiographic response to TMZ rechallenge in patients with recurrent anaplastic glioma and leptomeningeal dissemination. More severe leptomeningeal dissemination was observed on MRI in the present case compared with those in the study by Nandipati et al; however, neurological and radiological improvement was observed following chemotherapy with TMZ and bevacizumab for almost 10 weeks. We hypothesize that bevacizumab may help to normalize disrupted tumor vasculature and improve TMZ delivery, providing increased CNS penetration.

In conclusion, the current case report indicates that chemotherapy with TMZ and bevacizumab may result in short-term improvement of leptomeningeal dissemination of recurrent glioblastoma, with demonstrated neurological and radiological improvement. Further, large-scale studies are required to investigate the effect of chemotherapy regimens with TMZ and bevacizumab on patients with a wider variety of malignancies.

\section{Acknowledgements}

This work was supported by Grant-in-Aid for Scientific Research from the Ministry of Education, Science and Culture of Japan (grant no. 24791520 to Dr Yoshiko Okita).

\section{References}

1. Stupp R, Mason WP, van den Bent MJ, et al; European Organisation for Research and Treatment of Cancer Brain Tumor and Radiotherapy Groups; National Cancer Institute of Canada Clinical Trials Group: Radiotherapy plus concomitant and adjuvant temozolomide for glioblastoma. N Engl J Med 352: 987-996, 2005.

2. Lamborn KR, Yung WK, Chang SM, et al; North American Brain Tumor Consortium: Progression-free survival: an important end point in evaluating therapy for recurrent high-grade gliomas. Neuro Oncol 10: 162-170, 2008.

3. Wong ET, Hess KR, Gleason MJ, et al: Outcomes and prognostic factors in recurrent glioma patients enrolled onto phase II clinical trials. J Clin Oncol 17: 2572-2578, 1999.

4. Arita N, Taneda M and Hayakawa T: Leptomeningeal dissemination of malignant gliomas. Incidence, diagnosis and outcome. Acta Neurochir (Wien) 126: 84-92, 1994.

5. Awad I, Bay JW and Rogers L: Leptomeningeal metastasis from supratentorial malignant gliomas. Neurosurgery 19 247-251, 1986.

6. Vertosick FT Jr. and Selker RG: Brain stem and spinal metastases of supratentorial glioblastoma multiforme: a clinical series. Neurosurgery 27: 516-522, 1990.

7. Delattre JY, Walker RW and Rosenblum MK: Leptomeningeal gliomatosis with spinal cord or cauda equina compression: a complication of supratentorial gliomas in adults. Acta Neurol Scand 79: 133-139, 1989.
8. Nandipati S and Demopoulos A: Leptomeningeal dissemination of anaplastic glioma: prolonged survival in two patients treated with temozolomide. J Neurooncol 105: 663-665, 2011.

9. Narita Y: Drug review: Safety and efficacy of bevacizumab for glioblastoma and other brain tumors. Jpn J Clin Oncol 43: 587-595, 2013.

10. Groves MD, DeGroot J, Tremont I, Forman A, Kang S, Pei BL, et al: A pilot study of systemically administered bevacizumab with neoplastic meningitis NM: imaging, clinical, CSF, and biomarker outcomes. Neuro Oncol (OT-02) 13: iii85-iii91, 2011.

11. Fiorentino A, Caivano R, Chiumento C, Cozzolino M and Fusco V: Radiotherapy and bevacizumab for intramedullary and leptomenigeal metastatic glioblastoma: a case report and review of the literature. Int J Neurosci 122: 691-694, 2012

12. Hübner F, Braun V and Richter HP: Case reports of symptomatic metastases in four patients with primary intracranial gliomas. Acta Neurochir (Wien) 143: 25-29, 2001.

13. Onda K, Tanaka R, Takahashi H, Takeda N and Ikuta F: Cerebral glioblastoma with cerebrospinal fluid dissemination: a clinicopathological study of 14 cases examined by complete autopsy. Neurosurgery 25: 533-540, 1989.

14. Chamberlain MC: Combined-modality treatment of leptomeningeal gliomatosis. Neurosurgery 52: 324-330, 2003.

15. Miller K, Wang M, Gralow J, et al: Paclitaxel plus bevacizumab versus paclitaxel alone for metastatic breast cancer. N Engl J Med 357: 2666-2676, 2007.

16. Yang JC, Haworth L, Sherry RM, et al: A randomized trial of bevacizumab, an anti-vascular endothelial growth factor antibody, for metastatic renal cancer. N Engl J Med 349: 427-434, 2003.

17. Giantonio BJ, Catalano PJ, Meropol NJ, et al; Eastern Cooperative Oncology Group Study E3200: Bevacizumab in combination with oxaliplatin, fluorouracil, and leucovorin (FOLFOX4) for previously treated metastatic colorectal cancer: results from the Eastern Cooperative Oncology Group Study E3200. J Clin Oncol 25: 1539-1544, 2007.

18. Hurwitz H, Fehrenbacher L, Novotny W, et al: Bevacizumab plus irinotecan, fluorouracil, and leucovorin for metastatic colorectal cancer. N Engl J Med 350: 2335-2342, 2004.

19. Sandler A, Gray R, Perry MC, et al: Paclitaxel-carboplatin alone or with bevacizumab for non-small-cell lung cancer. N Engl J Med 355: 2542-2550, 2006

20. Vincent A, Lesser G, Brown D, et al: Prolonged regression of metastatic leptomeningeal breast cancer that has failed conventional therapy: a case report and review of the literature. J Breast Cancer 16: 122-126, 2013.

21. De Braganca KC, Janjigian YY, Azzoli CG, et al: Efficacy and safety of bevacizumab in active brain metastases from non-small cell lung cancer. J Neurooncol 100: 443-447, 2010.

22. Ku GY, Krol G and Ilson DH: Successful treatment of leptomeningeal disease in colorectal cancer with a regimen of bevacizumab, temozolomide, and irinotecan. J Clin Oncol 25: e14-e16, 2007.

23. Le Rhun E, Taillibert S and Chamberlain MC: Carcinomatous meningitis: Leptomeningeal metastases in solid tumors. Surg Neurol Int 4: S265-S288, 2013.

24. Mohan A and Ponnusankar S: Newer therapies for the treatment of metastatic breast cancer: a clinical update. Indian J Pharm Sci 75: 251-261, 2013.

25. Foubert F, Matysiak-Budnik T and Touchefeu Y: Options for metastatic colorectal cancer beyond the second line of treatment. Dig Liver Dis 46: 105-112, 2014.

26. Folkman J: Angiogenesis: an organizing principle for drug discovery? Nat Rev Drug Discov 6: 273-286, 2007.

27. Jain RK: Normalization of tumor vasculature: an emerging concept in antiangiogenic therapy. Science 307: 58-62, 2005.

28. Jain RK, Duda DG, Clark JW and Loeffler JS: Lessons from phase III clinical trials on anti-VEGF therapy for cancer. Nat Clin Pract Oncol 3: 24-40, 2006.

29. Chamberlain MC and Johnston SK: Salvage therapy with single agent bevacizumab for recurrent glioblastoma. J Neurooncol 96: 259-269, 2010.

30. Wong ET, Gautam S, Malchow C, Lun M, Pan E and Brem S: Bevacizumab for recurrent glioblastoma multiforme: a meta-analysis. J Natl Compr Canc Netw 9: 403-407, 2011. 